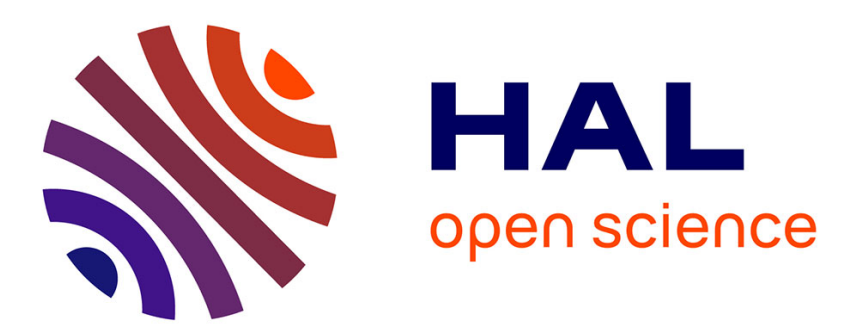

\title{
Multilevel stochastic reduced-order model in linear structural dynamics for complex structures
}

Olivier Ezvan, Anas Batou, Christian Soize

\section{To cite this version:}

Olivier Ezvan, Anas Batou, Christian Soize. Multilevel stochastic reduced-order model in linear structural dynamics for complex structures. ECCOMAS 2016, 7th European Congress on Computational Methods in Applied Sciences and Engineering, Jun 2016, The Island of Crete, Greece. pp.1-12. hal01353218

\section{HAL Id: hal-01353218 \\ https://hal.science/hal-01353218}

Submitted on 10 Aug 2016

HAL is a multi-disciplinary open access archive for the deposit and dissemination of scientific research documents, whether they are published or not. The documents may come from teaching and research institutions in France or abroad, or from public or private research centers.
L'archive ouverte pluridisciplinaire HAL, est destinée au dépôt et à la diffusion de documents scientifiques de niveau recherche, publiés ou non, émanant des établissements d'enseignement et de recherche français ou étrangers, des laboratoires publics ou privés. 


\title{
MULTILEVEL STOCHASTIC REDUCED-ORDER MODEL IN LINEAR STRUCTURAL DYNAMICS FOR COMPLEX STRUCTURES
}

\author{
O. Ezvan $^{1}$, A. Batou ${ }^{1}$, and C. Soize ${ }^{1}$ \\ ${ }^{1}$ Université Paris-Est, Laboratoire Modélisation et Simulation Multi-Echelle, \\ MSME UMR 8208 CNRS \\ 5 bd Descartes, 77454 Marne-la-Vallée, France \\ e-mail: \{olivier.ezvan, anas.batou, christian.soize\}@univ-paris-est.fr
}

Keywords: Uncertainty quantification, Reduced-Order Model, Structural dynamics

\begin{abstract}
We present the construction of a multilevel stochastic reduced-order model devoted to the robust prediction of frequency response functions of complex linear dynamical systems that are characterized by the presence of several structural scales in which there are local displacements in addition to the usual global displacements, and which are associated with the distinct low-, medium-, and high-frequency bands. As the levels of uncertainties are different in the three frequency bands, a multilevel stochastic reduced-order model using several orthogonal subspaces associated with the several types of displacements is developed. The objective of the paper is to demonstrate the capability of the multilevel stochastic reduced-order model to adapt the stochastic modeling of uncertainties to each one of the three frequency bands.
\end{abstract}




\section{Introduction}

We present a new method for the robust prediction of frequency response functions (FRF) of complex linear dynamical structures exhibiting a high modal density. Commonly, the low-frequency range [1] is characterized by the presence of a few dozen isolated eigenfrequencies that are associated with global modes, in which case the modal analysis method [2, 3, 4] allows an effective and efficient small-dimension reduced-order model (ROM) to be obtained. In contrast, the complex structures we deal with can exhibit more than hundreds or thousands modes in low frequencies. This unusual feature is due to the presence of several structural scales within the complex geometry of the structure. Small flexible components attached to the stiff skeleton of the structure induce the presence of numerous local modes intertwined with the usual global modes of the stiff skeleton. For such complex structures, besides the absence of separation of scales, the global displacements (or global modes) cannot easily be identified because coupled with the large-amplitude local displacements (or local modes). For constructing an adapted reduced-order model of such complex structures, a first version of a two-levels reduced-order model has been proposed [5], and then has been extended and applied to complex mechanical systems [6, 7]. Then the methodology has been generalized [8] for constructing multilevel reduced-order models. This paper is mainly devoted to the stochastic aspects and their implementation in the multilevel reduced-order model.

First, the proposed method allows for constructing a ROM of smaller dimension, which is obtained by introducing a subspace of global displacements. The construction of the latter is based on the introduction of high-degree polynomial shape functions. The vector basis of the global-displacements subspace is constituted of the eigenmodes calculated using such an approximation for the kinetic energy. The choice of the polynomial degree allows for controlling the filtering between the so-called global and local displacements, as well as the resulting dimension and accuracy of the so-called global ROM. Furthermore, it is well known that local displacements are in general more sensitive to uncertainties than global displacements. The nonparametric probabilistic approach [9] allows all sources of uncertainty to be globally accounted for by randomizing each reduced matrix whose probability density function, constructed applying the maximum entropy principle [10, 11], is parameterized by a unique dispersion hyperparameter. In order to separately control the uncertainty level of the displacements of each of the scales, we propose to use a multilevel ROM, based on the introduction of orthogonal subspaces. The basis of each of these subspaces is constructed by using, notably, the aforementioned polynomial approximation for the kinetic energy, with an adapted polynomial degree. Each basis is constituted of displacements associated with a given structural scale. Then, the multilevel stochastic ROM is obtained by using the nonparametric probabilistic approach for each scale. This stochastic model is then controlled by some dispersion hyperparameters devoted to each scale. The method is applied to the complex computational model of a car. The objective of this work is to present a sensitivity analysis of the multilevel stochastic ROM with respect to the 
dispersion hyperparameters in order to demonstrate the capability of the method proposed to adapt the stochastic modeling to the level of uncertainties as a function of the frequency bands.

\section{Classical nominal reduced-order model}

Let $[\mathbb{M}],[\mathbb{D}]$, and $[\mathbb{K}]$ denote the $(m \times m)$ positive-definite real symmetric mass, damping and stiffness matrices of a $m$-dimensional finite element model of a linear damped structure with bounded domain, $\Omega$. The $m$-dimensional complex vector $\mathbb{U}(\omega)$ of the displacements satisfies, for all $\omega$ in the frequency band $\mathcal{B}$, the matrix equation,

$$
\left(-\omega^{2}[\mathbb{M}]+i \omega[\mathbb{D}]+[\mathbb{K}]\right) \mathbb{U}(\omega)=\mathbb{F}(\omega),
$$

in which the $m$-dimensional complex vector $\mathbb{F}(\omega)$ represents the external forces. For $\alpha=1, \ldots, m$, the elastic modes $\varphi_{\alpha}$ are the eigenvectors of the following generalized eigenvalue problem associated with the conservative dynamical system,

$$
[\mathbb{K}] \boldsymbol{\varphi}_{\alpha}=\lambda_{\alpha}[\mathbb{M}] \boldsymbol{\varphi}_{\alpha}
$$

in which $\lambda_{\alpha}$ is the eigenvalue associated with mode $\varphi_{\alpha}$ such that $\lambda_{\alpha}=\omega_{\alpha}^{2}$ and $\omega_{\alpha}=$ $2 \pi f_{\alpha}$, with $f_{\alpha}$ the associated eigenfrequency in Hz. The classical nominal reducedorder model (C-NROM) is obtained by projecting Eq. (1) onto the subspace, $\mathcal{S}_{c} \subset \mathbb{C}^{m}$, spanned by the first $n$ elastic modes (associated with the $n$ smallest eigenfrequencies). Normalizing the eigenmodes with respect to the mass matrix, the associated reducedorder basis $(\mathrm{ROB})$ is given by $[\Phi]=\left[\boldsymbol{\varphi}_{1} \ldots \boldsymbol{\varphi}_{n}\right]$ and verifies $[\Phi]^{T}[\mathbb{M}][\Phi]=\left[I_{n}\right]$ as well as $[\Phi]^{T}[\mathbb{K}][\Phi]=[\Lambda]$, with $[\Lambda]$ the matrix of the first $n$ eigenvalues. In the rest of this paper, the eigenvectors of any eigenvalue problem such as in Eq. (2) will follow the same normalization. Introducing the generalized damping matrix $[\mathcal{D}]=$ $[\Phi]^{T}[\mathbb{D}][\Phi]$ and the vector $\mathcal{F}(\omega)=[\Phi]^{T} \mathbb{F}(\omega)$ of the generalized forces, the vector $\mathbf{q}(\omega)=\left(q_{1}(\omega) \ldots q_{n}(\omega)\right)$ of the generalized coordinates of the C-NROM is the solution of the reduced-matrix equation,

$$
\left(-\omega^{2}\left[I_{n}\right]+i \omega[\mathcal{D}]+[\Lambda]\right) \mathbf{q}(\omega)=\mathcal{F}(\omega),
$$

and allows displacements $\mathbb{U}(\omega)$ to be approximated by

$$
\mathbb{U}(\omega) \simeq[\Phi] \mathbf{q}(\omega)=\sum_{\alpha=1}^{n} q_{\alpha}(\omega) \boldsymbol{\varphi}_{\alpha}
$$

In the rest of this paper, several ROMs will be constructed. For simplicity, the notations will be introduced only for the associated ROB, since any ROM is straightforwardly obtained, using its ROB, by projection of Eq. (1) and by recovering the physical degrees of freedom (DOF) $\mathbb{U}(\omega)$ such as in Eq. (4).

For the complex structures under consideration, due to the presence of numerous local displacements, dimension $n$ of the C-NROM is quite large. We propose the 
construction of a global-displacements ROB, from which local displacements are removed. Such filtering relies on the introduction of a reduced kinematics for the mass matrix.

\section{Reduced-kinematics mass matrix}

The proposed filtering of local displacements is based on the use of polynomial shape functions defined on domain $\Omega$, the filtering being controlled by the degree, $\mathcal{D}$, of the polynomial approximation. The construction of the polynomial basis is detailed in [8]. Let $[B]$ denote the $\left(m \times N_{p}\right)$ real matrix constituted of the concatenation of the $N_{p}=(\mathcal{D}+1)(\mathcal{D}+2)(\mathcal{D}+3) / 2$ polynomials, which are orthogonal such that

$$
[B]^{T}[\mathbb{M}][B]=\left[I_{N_{p}}\right] .
$$

The polynomials are only used for approximating the kinetic energy (the elastic energy remains exact). Let $\mathbb{V}(t)$ denote a time-dependent real vector of dimension $m$. The orthogonal projection $\mathbb{V}^{r}(t)$ of $\mathbb{V}(t)$ onto the subspace spanned by the polynomials basis is given by

$$
\mathbb{V}^{r}(t)=[\mathbb{P}] \mathbb{V}(t),
$$

in which the projector $[\mathbb{P}]$ is a $(m \times m)$ real matrix which is written $[8]$ as

$$
[\mathbb{P}]=[B][B]^{T}[\mathbb{M}]
$$

Then, the kinetic energy $E_{k}(\mathbb{V}(t))=\frac{1}{2} \mathbb{V}(t)^{T}[\mathbb{M}] \mathbb{V}(t)$ is replaced by the reduced kinetic energy $E_{k}^{r}(\mathbb{V}(t))=\frac{1}{2} \mathbb{V}^{r}(t)^{T}[\mathbb{M}] \mathbb{V}^{r}(t)=\frac{1}{2} \mathbb{V}(t)^{T}\left[\mathbb{M}^{r}\right] \mathbb{V}(t)$ in which the $(m \times m)$ reduced-kinematics mass matrix $\left[\mathbb{M}^{r}\right]$ is positive-semidefinite symmetric real of rank $N_{p}$ and such that

$$
\left[\mathbb{M}^{r}\right]=[\mathbb{M}][B][B]^{T}[\mathbb{M}]
$$

For constructing the global-displacements $\mathrm{ROB}$, matrix $\left[\mathrm{M}^{r}\right]$ is used instead of $[\mathbb{M}]$.

\section{Global-displacements reduced-order basis}

\subsection{Definition}

Let $\psi_{\alpha}^{g}\left(\alpha=1, \ldots, N_{p}\right)$ be the eigenvectors and let $\sigma_{\alpha}^{g}$ be the associated eigenvalues such that

$$
[\mathbb{K}] \boldsymbol{\psi}_{\alpha}^{g}=\sigma_{\alpha}^{g}\left[\mathbb{M}^{r}\right] \boldsymbol{\psi}_{\alpha}^{g}
$$

for which the kinetic energy is subjected to the polynomial approximation and which allows the $(m \times \nu)$ real matrix $\left[\Psi^{g}\right]=\left[\boldsymbol{\psi}_{1}^{g} \ldots \boldsymbol{\psi}_{\nu}^{g}\right]$ to be constructed, with $\nu \leq N_{p}$. Then, the ROB associated with the global nominal reduced-order model (G-NROM) is 
given by $\left[\Phi^{g}\right]=\left[\Psi^{g}\right][R]$, where $[R]=\left[\mathbf{r}_{1} \ldots \mathbf{r}_{n_{g}}\right]$ is constituted of the $n_{g}$ eigenvectors $\mathbf{r}_{\alpha}$ of the small-dimension generalized eigenvalue problem

$$
\left(\left[\Psi^{g}\right]^{T}[\mathbb{K}]\left[\Psi^{g}\right]\right) \mathbf{r}_{\alpha}=\lambda_{\alpha}^{g}\left(\left[\Psi^{g}\right]^{T}[\mathbb{M}]\left[\Psi^{g}\right]\right) \mathbf{r}_{\alpha}
$$

where dimension $n_{g}$ of the G-NROM is chosen as minimum under the constraint $f_{n_{g}}^{g} \geq$ $f^{c}$, with $f_{\alpha}^{g}=\sqrt{\lambda_{\alpha}^{g}} / 2 \pi$. Frequency $f^{c}$ is related to the upper bound of frequency band of analysis $\mathcal{B}$. The global-displacements subspace, spanned by the $n_{g}$ columns of $\left[\Phi^{g}\right]$, is denoted by $\mathcal{S}_{g}$. The matrix $\left[\Lambda^{g}\right]$ of the associated eigenvalues is such that $\left[\Lambda^{g}\right]=\left[\Phi^{g}\right]^{T}[\mathbb{K}]\left[\Phi^{g}\right]$.

\subsection{Numerical implementation}

The proposed method is adapted so as to be non-intrusive with respect to commercial software for which extraction of mass and stiffness matrices can be difficult or even impossible. Given the considered approximation for constructing mass matrix $\left[\mathbb{M}^{r}\right]$, replacing consistent mass matrix $[\mathbb{M}]$ by a lumped approximation in order to construct $\left[\mathbb{M}^{r}\right]$ is legitimate. We then propose an indirect method for constructing the global-displacements ROB.

Let $\mathcal{S}_{0}$ be a subspace spanned by the $n_{0}$ columns of a ROB $\left[\Phi^{0}\right]$, which can, for instance, be constituted of elastic modes, available through commercial software. We suppose having $\left[\Phi^{0}\right]^{T}[\mathbb{M}]\left[\Phi^{0}\right]=\left[I_{n_{0}}\right]$ and $\left[\Phi^{0}\right]^{T}[\mathbb{K}]\left[\Phi^{0}\right]=\left[\Lambda^{0}\right]$ with $\left[\Lambda^{0}\right]$ a diagonal matrix with positive elements. Then, considering the approximation $\mathcal{S}_{g} \subseteq \mathcal{S}_{0}$ obtained writing $\psi_{\alpha}^{g}=\left[\Phi^{0}\right] \mathbf{s}_{\alpha}$ for all $\alpha=1, \ldots, N_{p}$, Eq. (9) leads us to the reduced-order generalized eigenvalue problem

$$
\left(\left[\Phi^{0}\right]^{T}[\mathbb{K}]\left[\Phi^{0}\right]\right) \mathbf{s}_{\alpha}=\sigma_{\alpha}^{g}\left(\left[\Phi^{0}\right]^{T}\left[\mathbb{M}^{r}\right]\left[\Phi^{0}\right]\right) \mathbf{s}_{\alpha}
$$

Since $\left[\Phi^{0}\right]^{T}[\mathbb{K}]\left[\Phi^{0}\right]=\left[\Lambda^{0}\right]$, the latter eigenvalue problem does not require having $[\mathbb{K}]$ but $\left[\Lambda^{0}\right]$ instead. Moreover, denoting as $\left[\mathbb{M}^{\ell}\right]$ a lumped approximation of $[\mathbb{M}]$, the generalized mass matrix of Eq. (11) is approximated as

$$
\left[\Phi^{0}\right]^{T}\left[\mathbb{M}^{r}\right]\left[\Phi^{0}\right] \simeq\left[N^{0}\right]\left[N^{0}\right]^{T}
$$

with $\left[N^{0}\right]=\left[\Phi^{0}\right]^{T}\left[\mathbb{M}^{\ell}\right]\left[B^{\ell}\right]$, in which $\left[B^{\ell}\right]$ denotes the orthonormalization of $[B]$ with respect to $\left[\mathbb{M}^{\ell}\right]$. Matrix $[S]=\left[\mathbf{s}_{1} \ldots \mathbf{s}_{\nu}\right]$ is such that $\left[\Psi^{g}\right]=\left[\Phi^{0}\right][S]$ and we then have $\left[\Phi^{g}\right]=\left[\Phi^{0}\right]\left[Q^{g}\right]$ with $\left[Q^{g}\right]=[S][R]$. Furthermore, reduced matrices involved in Eq. (10) are given by

$$
\left[\Psi^{g}\right]^{T}[\mathbb{K}]\left[\Psi^{g}\right]=\left[\Sigma^{g}\right], \quad\left[\Psi^{g}\right]^{T}[\mathbb{M}]\left[\Psi^{g}\right]=[S]^{T}[S]
$$

with $\left[\Sigma^{g}\right]$ the matrix of the first $\nu$ eigenvalues $\sigma_{\alpha}^{g}$. 


\subsection{Complementary local-displacements reduced-order basis}

The complementary basis of local displacements belonging to subspace $\mathcal{S}_{0}$ spans a subspace $\mathcal{S}_{\ell}$ that is such that

$$
\mathcal{S}_{0}=\mathcal{S}_{g} \oplus \mathcal{S}_{\ell}
$$

which means that $\mathcal{S}_{g}$ and $\mathcal{S}_{\ell}$ are in direct sum. The construction of the associated ROB, $\left[\Phi^{\ell}\right]$, is proposed as follows. It verifies $\left[\Phi^{g}\right]^{T}[\mathbb{M}]\left[\Phi^{\ell}\right]=[0]$ (orthogonality of the local basis vectors, $\varphi_{\alpha}^{\ell}$, and the global basis vectors, $\varphi_{\alpha}^{g}$, with respect to the mass matrix) as well as $\left[\Phi^{\ell}\right]=\left[\Phi^{0}\right]\left[Q^{\ell}\right]$ (the local basis vectors belong to $\mathcal{S}_{0}$ ), with $\left[Q^{\ell}\right]$ a real matrix of generalized coordinates that is defined hereinafter. The orthogonality condition is written as $[C]\left[Q^{\ell}\right]=[0]$, where matrix $[C]$ is given by $[C]=\left[\Phi^{g}\right]^{T}[\mathbb{M}]\left[\Phi^{0}\right]$, and can be rewritten as

$$
[C]=\left[Q^{g}\right]^{T}
$$

Let $[C]=\left[U_{C}\right]\left[\Sigma_{C}\right]\left[V_{C}\right]^{T}$ be the singular value decomposition (SVD) of $[C]$. Let $[Z]$ be the concatenation of the columns of $\left[V_{C}\right]$ associated with the zero singular values (zero diagonal elements of $\left[\Sigma_{C}\right]$ ). Then, the columns of $[Z]$ constitute a vector basis of the kernel of $[C]$ and matrix $[Z]$ verifies $[Z]^{T}[Z]=\left[I_{n_{\ell}}\right]$, where $n_{\ell}=n_{0}-n_{g}$ is the dimension of the kernel of $[C]$. Orthogonality condition $[C]\left[Q^{\ell}\right]=[0]$ leads us to $\left[Q^{\ell}\right]=[Z][U]$, with $[U]$ a matrix of generalized coordinates. The columns $\mathbf{u}_{\alpha}$ of $[U]=\left[\mathbf{u}_{1} \ldots \mathbf{u}_{n_{\ell}}\right]$ are the solutions of the reduced-order generalized eigenvalue problem

$$
\left([Z]^{T}\left[\Phi^{0}\right]^{T}[\mathbb{K}]\left[\Phi^{0}\right][Z]\right) \mathbf{u}_{\alpha}=\lambda_{\alpha}^{\ell}\left([Z]^{T}\left[\Phi^{0}\right]^{T}[\mathbb{M}]\left[\Phi^{0}\right][Z]\right) \mathbf{u}_{\alpha}
$$

which can be more simply rewritten as the eigenvalue problem

$$
\left([Z]^{T}\left[\Lambda^{0}\right][Z]\right) \mathbf{u}_{\alpha}=\lambda_{\alpha}^{\ell} \mathbf{u}_{\alpha}
$$

\section{Multilevel nominal and stochastic reduced-order models}

To sum up, for a given polynomial degree $\mathcal{D}$ and a given truncation order $\nu$ for $\left[\Psi^{g}\right]=\left[\boldsymbol{\psi}_{1}^{g} \ldots \boldsymbol{\psi}_{\nu}^{g}\right]$, global-displacements subspace $\mathcal{S}_{g}$ and local-displacements subspace $\mathcal{S}_{\ell}$ of any given space $\mathcal{S}_{0}$ can be constructed such that $\mathcal{S}_{0}=\mathcal{S}_{g} \oplus \mathcal{S}_{\ell}$. Dimension $n_{g} \leq \nu$ of $\mathcal{S}_{g}$ also depends on cutting frequency $f^{c}$. Therefore, there is a function $\mathcal{G}$ such that

$$
\mathcal{G}:\left(\mathcal{S}_{0}, \mathcal{D}, \nu, f^{c}\right) \longmapsto\left(\mathcal{S}_{g}, \mathcal{S}_{\ell}\right)
$$

which includes the described construction in a compact form. 


\subsection{Definition of the orthogonal subspaces}

For the multilevel nominal reduced-order model (ML-NROM), three subspaces $\mathcal{S}_{\mathcal{L}}$, $\mathcal{S}_{\mathcal{M}}$, and $\mathcal{S}_{\mathcal{H}}$ associated with the low-, medium-, and high-frequency bands (LF, MF, and HF) are constructed. These subspaces are such that $\mathcal{S}_{g}=\mathcal{S}_{\mathcal{L}} \oplus \mathcal{S}_{\mathcal{M}} \oplus \mathcal{S}_{\mathcal{H}}$. Space $\mathcal{S}_{g}$ corresponds to a filtering of local displacements that allows the final dimension (and thus the computational cost) of the ML-NROM to be reduced. Subspaces $\mathcal{S}_{g}$ and $\mathcal{S}_{\ell}$ are given by

$$
\left(\mathcal{S}_{g}, \mathcal{S}_{\ell}\right)=\mathcal{G}\left(\mathcal{S}_{c}, \mathcal{D}_{g}, \nu_{g}, f_{g}^{c}\right)
$$

where we recall space $\mathcal{S}_{c}$ to be spanned by the first $n$ elastic modes that would be used in a classical modal analysis, with $f_{g}^{c}$ a cutting frequency associated with the upper bound of frequency band of analysis $\mathcal{B}$, and where parameters $\mathcal{D}_{g}$ and $\nu_{g}$ allow the filtering to be controlled. It should be noted that, for constructing the ML-NROM, the construction of $\mathcal{S}_{\ell}$ that is such that $\mathcal{S}_{c}=\mathcal{S}_{g} \oplus \mathcal{S}_{\ell}$, is not needed.

We then introduce a second filtering, using $\mathcal{D}_{\mathcal{L M}} \leq \mathcal{D}_{g}$ and $\nu_{\mathcal{L M}} \leq n_{g}$ as well as a cutting frequency $f_{\mathcal{L M}}^{c}$ associated with the upper bound of the MF band. The associated global-displacements and local-displacements subspaces $\mathcal{S}_{\mathcal{L} \mathcal{M}}$ and $\mathcal{S}_{\mathcal{H}}$ are given by

$$
\left(\mathcal{S}_{\mathcal{L M}}, \mathcal{S}_{\mathcal{H}}\right)=\mathcal{G}\left(\mathcal{S}_{g}, \mathcal{D}_{\mathcal{L M}}, \nu_{\mathcal{L M}}, f_{\mathcal{L} \mathcal{M}}^{c}\right)
$$

and satisfy $\mathcal{S}_{g}=\mathcal{S}_{\mathcal{L M}} \oplus \mathcal{S}_{\mathcal{H}}$.

For obtaining subspaces $\mathcal{S}_{\mathcal{L}}$ and $\mathcal{S}_{\mathcal{M}}$, a third filtering parameterized by $\mathcal{D}_{\mathcal{L}} \leq \mathcal{D}_{\mathcal{L M}}$ and $\nu_{\mathcal{L}} \leq \operatorname{dim}\left(\mathcal{S}_{\mathcal{L M}}\right)$ as well as a cutting frequency $f_{\mathcal{L}}^{c}$ associated with the upper bound of the LF band is introduced. The associated global-displacements and localdisplacements subspaces $\mathcal{S}_{\mathcal{L}}$ and $\mathcal{S}_{\mathcal{M}}$ are given by

$$
\left(\mathcal{S}_{\mathcal{L}}, \mathcal{S}_{\mathcal{M}}\right)=\mathcal{G}\left(\mathcal{S}_{\mathcal{L} \mathcal{M}}, \mathcal{D}_{\mathcal{L}}, \nu_{\mathcal{L}}, f_{\mathcal{L}}^{c}\right)
$$

Since $\mathcal{S}_{\mathcal{L M}}=\mathcal{S}_{\mathcal{L}} \oplus \mathcal{S}_{\mathcal{M}}$, we thus have $\mathcal{S}_{g}=\mathcal{S}_{\mathcal{L}} \oplus \mathcal{S}_{\mathcal{M}} \oplus \mathcal{S}_{\mathcal{H}}$.

\subsection{Multilevel nominal reduced-order model}

Due to the orthogonality property, it can be shown that the reduced mass matrix of the ML-NROM, $[M]$, is such that $[M]=\left[I_{n_{g}}\right]$. In contrast, the reduced stiffness matrix $[K]$ of the ML-NROM is a full matrix that is written as

$$
[K]=\left[\begin{array}{lll}
{\left[K^{\mathcal{L L}}\right]} & {\left[K^{\mathcal{L M}}\right]} & {\left[K^{\mathcal{L H}}\right]} \\
{\left[K^{\mathcal{M L}}\right]} & {\left[K^{\mathcal{M M}}\right]} & {\left[K^{\mathcal{M H}}\right]} \\
{\left[K^{\mathcal{H L}}\right]} & {\left[K^{\mathcal{H M}}\right]} & {\left[K^{\mathcal{H H}}\right]}
\end{array}\right],
$$

in which, for $\mathcal{I}$ and $\mathcal{J}$ in $\{\mathcal{L}, \mathcal{M}, \mathcal{H}\}$, the matrix block $\left[K^{\mathcal{I J}}\right]$ is given by $\left[K^{\mathcal{I J}}\right]=$ $\left[\Phi^{\mathcal{I}}\right]^{T}[\mathbb{K}]\left[\Phi^{\mathcal{J}}\right]$, with $\left[\Phi^{\mathcal{I}}\right]$ the ROB of $\mathcal{S}_{\mathcal{I}}$. The damping matrix $[D]$ of the ML-NROM is also a full matrix with the blocks $\left[D^{\mathcal{I} J}\right]=\left[\Phi^{\mathcal{I}}\right]^{T}[\mathbb{D}]\left[\Phi^{\mathcal{J}}\right]$. 


\subsection{Multilevel stochastic reduced-order model}

The construction of the multilevel stochastic reduced-order model (ML-SROM) is based on the nonparametric probabilistic approach of uncertainties. This approach consists in replacing any positive-definite symmetric real $(N \times N)$ matrix $[X]$ of a $\mathrm{ROM}$ by an associated random matrix $[\mathrm{X}]$ whose probability density function is obtained applying the maximum entropy principle under the constraints (available information)

- Matrix $[\mathbf{X}]$ is with values in the set of all the positive-definite symmetric real $(N \times N)$ matrices.

- $E\{[\mathbf{X}]\}=[X]$, where $E$ is the mathematical expectation: the mean value is chosen as the value of the nominal model.

- $E\left\{\left\|[\mathbf{X}]^{-1}\right\|_{F}^{2}\right\}<+\infty$, with $\|.\|_{F}$ denoting the Frobenius norm.

Given the Cholesky factorization $[X]=\left[L_{X}\right]^{T}\left[L_{X}\right]$ with $\left[L_{X}\right]$ an upper triangular matrix, random matrix $[\mathbf{X}]$ is written as

$$
[\mathbf{X}]=\left[L_{X}\right]^{T}[\mathbf{G}]\left[L_{X}\right],
$$

in which the algebraic construction of the positive-definite symmetric real $(N \times N)$ random matrix $[\mathbf{G}]$ is given in [9] and only depends on a scalar (dispersion) hyperparameter, $\delta$, verifying

$$
\delta^{2}=\frac{1}{N} E\left\{\|[\mathbf{G}]-\left.\left[I_{N}\right]\right|_{F} ^{2}\right\}
$$

In general, the local displacements are more sensitive to uncertainties than the global displacements. The ML-SROM allows the variability of each type of displacements (belonging to either subspace $\mathcal{S}_{\mathcal{L}}$, subspace $\mathcal{S}_{\mathcal{M}}$, or subspace $\mathcal{S}_{\mathcal{H}}$ ) to be modeled separately. For each random matrix $[\mathbf{A}]=[\mathbf{M}],[\mathbf{D}],[\mathbf{K}]$ of the ML-SROM, three dispersion hyperparameters $\delta_{\mathcal{L}}^{A}, \delta_{\mathcal{M}}^{A}$, and $\delta_{\mathcal{H}}^{A}$ are thus introduced (with $A=M, D, K$ referring to each deterministic matrix of the ML-NROM). For $\mathcal{I}=\mathcal{L}, \mathcal{M}, \mathcal{H}$ and $A=M, D, K$, let $\left[\mathbf{G}_{\mathcal{I}}^{A}\right]$ denote the random matrix with the same construction as $[\mathbf{G}]$ and verifying $\left(\delta_{\mathcal{I}}^{A}\right)^{2}=\frac{1}{n_{\mathcal{I}}} E\left\{\left\|\left[\mathbf{G}_{\mathcal{I}}^{A}\right]-\left[I_{n_{\mathcal{I}}}\right]\right\|_{F}^{2}\right\}$, in which $n_{\mathcal{I}}=\operatorname{dim}\left(\mathcal{S}_{\mathcal{I}}\right)$. Given the Cholesky factorization $[A]=\left[L_{A}\right]^{T}\left[L_{A}\right]$ of each deterministic matrix $[A]$ of the ML-NROM, each random matrix $[\mathbf{A}]$ of the ML-SROM is defined as

$$
[\mathbf{A}]=\left[L_{A}\right]^{T}\left[\mathbf{G}^{A}\right]\left[L_{A}\right],
$$

in which the $\left(n_{g} \times n_{g}\right)$ positive-definite symmetric real random matrix $\left[\mathbf{G}^{A}\right]$ is given by

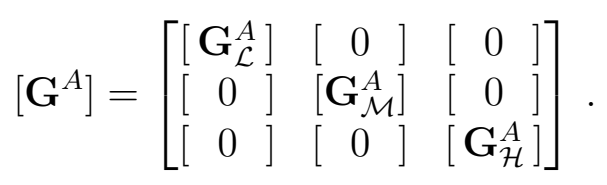


Denoting as $[\Psi]=\left[\left[\Phi^{\mathcal{L}}\right]\left[\Phi^{\mathcal{M}}\right]\left[\Phi^{\mathcal{H}}\right]\right]$ the ROB of the multilevel ROM and introducing the deterministic vector $\mathbf{f}(\omega)=[\Psi]^{T} \mathbb{F}(\omega)$ of generalized forces, the random generalized coordinates $\mathbf{Q}(\omega)=\left(Q_{1}(\omega) \ldots Q_{n_{g}}(\omega)\right)$ of the ML-SROM are the solutions of the small-dimension matrix equation

$$
\left(-\omega^{2}[\mathbf{M}]+i \omega[\mathbf{D}]+[\mathbf{K}]\right) \mathbf{Q}(\omega)=\mathbf{f}(\omega)
$$

which is solved using the Monte-Carlo simulation method [12], and allow the random response $\mathbf{U}(\omega)$ associated with $\mathbb{U}(\omega)$ to be obtained from

$$
\mathbf{U}(\omega)=[\Psi] \mathbf{Q}(\omega) .
$$

\section{Application to the complex computational model of a car}

\subsection{Nominal reduced-order models}

The nominal finite element model is used for constructing the multilevel stochastic ROM. The finite element model has $m=8,000,000$ DOF and the C-NROM is constructed using the first $n=16,192$ elastic modes as a ROB (we have $f_{n}=1,600 \mathrm{~Hz}$ ). Then, the global-displacements nominal ROM associated with subspace $\mathcal{S}_{g}$ is constructed choosing $\mathcal{D}_{g}=31, \nu=7,000$ and $f_{g}^{c}=1,525 \mathrm{~Hz}$. Follow the construction of subspaces $\mathcal{S}_{\mathcal{L M}}$ and $\mathcal{S}_{\mathcal{L}}$ obtained choosing $\mathcal{D}_{\mathcal{L M}}=15, \nu_{\mathcal{L M}}=1,950$, $f_{\mathcal{L} \mathcal{M}}^{c}=1,000 \mathrm{~Hz}$ and $\mathcal{D}_{\mathcal{L}}=4, \nu_{\mathcal{L}}=400, f_{\mathcal{L} \mathcal{M}}^{c}=300 \mathrm{~Hz}$. Dimensions $n_{g}, n_{\mathcal{L} \mathcal{M}}$ and $n_{\mathcal{L}}$ of subspaces $\mathcal{S}_{g}, \mathcal{S}_{\mathcal{L M}}$, and $\mathcal{S}_{\mathcal{L}}$ are $n_{g}=6,984, n_{\mathcal{L M}}=1,919$, and $n_{\mathcal{L}}=344$.

\subsection{Multilevel stochastic reduced-order model}

The objective of the stochastic model is to take into account the variability as well as the uncertainties in the computational model (due to both the model-parameter uncertainties and the modeling errors). The global displacements, which usually correspond to resonances in the low-frequency band, are more robust with respect to small design changes, variability, and model uncertainties. We recall the multilevel ROM to be constituted of the following three orthogonal subspaces:

- $\mathcal{S}_{\mathcal{L}}$, constituted of displacements obtained using degree $\mathcal{D}_{\mathcal{L}}=4$ for the kinetic energy and associated with the frequencies below $f_{\mathcal{L}}^{c}=300 \mathrm{~Hz}$, thus expected to consist in low-frequency global displacements.

- $\mathcal{S}_{\mathcal{M}}$, constituted of displacements obtained using degree $\mathcal{D}_{\mathcal{L M}}=15$ for the kinetic energy, associated with the frequencies below $f_{\mathcal{L} \mathcal{M}}^{c}=1,000 \mathrm{~Hz}$ and orthogonal to $\mathcal{S}_{\mathcal{L}}$, thus expected to consist in combinations of a few global displacements in presence of many local displacements in the low- and midfrequency bands.

- $\mathcal{S}_{\mathcal{H}}$, constituted of displacements obtained using degree $\mathcal{D}_{g}=31$ for the kinetic energy, associated with the frequencies below $f_{g}^{c}=1,525 \mathrm{~Hz}$ and orthogonal to both $\mathcal{S}_{\mathcal{L}}$ and $\mathcal{S}_{\mathcal{M}}$, thus expected to mainly consist in local displacements present 
throughout the entire band with an increasing density towards higher frequencies.

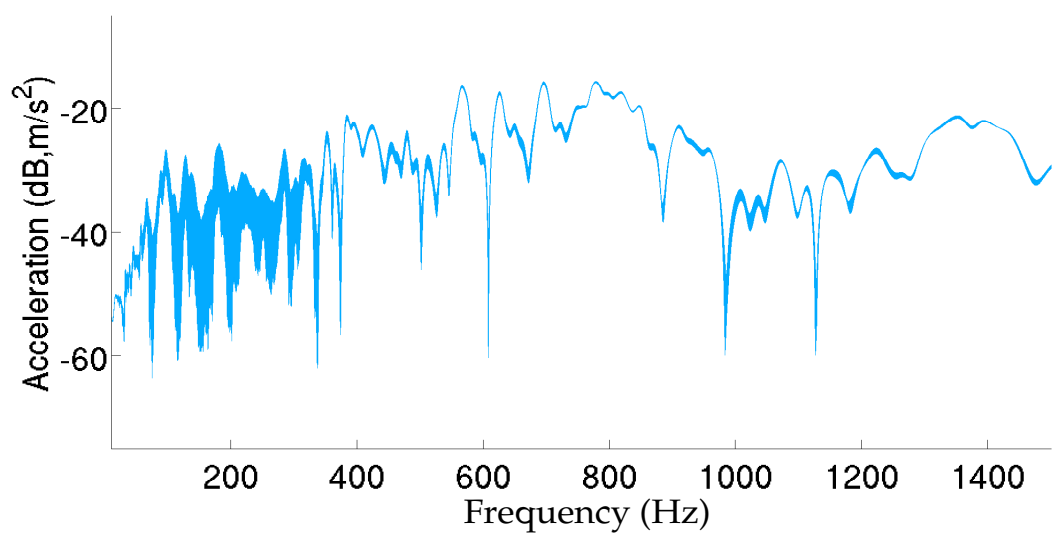

Figure 1: Random response using the ML-SROM with $\delta_{\mathcal{L}}^{M}=0.1$ and for all other dispersion hyperparameters set to zero ( $95 \%$ confidence interval).

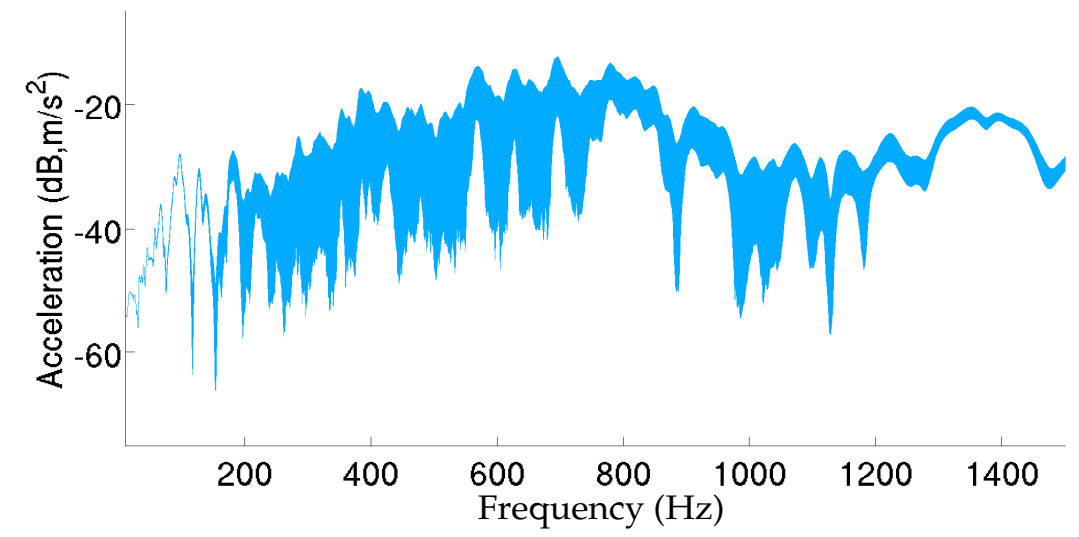

Figure 2: Random response using the ML-SROM with $\delta_{\mathcal{M}}^{M}=0.1$ and for all other dispersion hyperparameters set to zero ( $95 \%$ confidence interval).

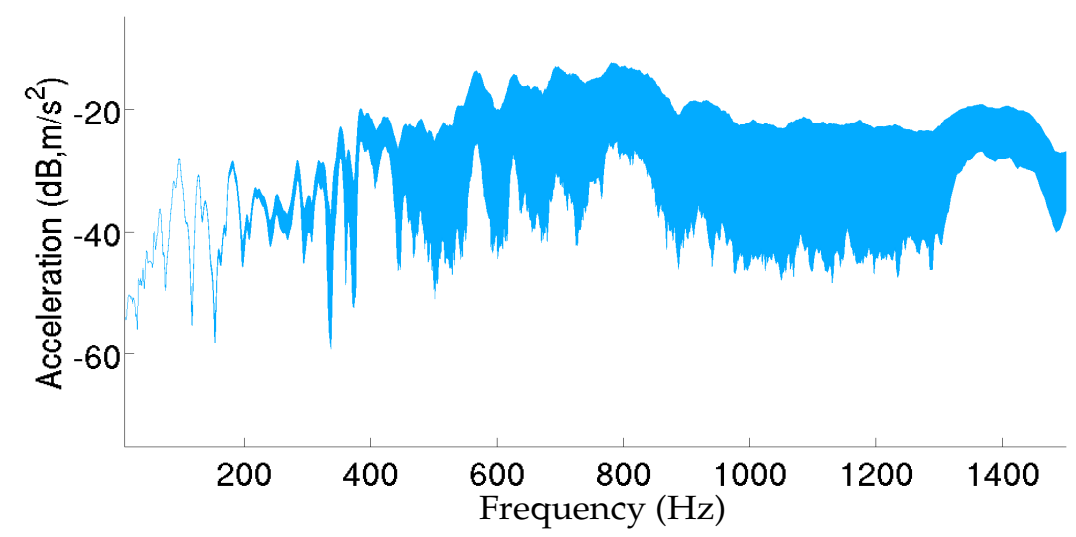

Figure 3: Random response using the ML-SROM with $\delta_{\mathcal{H}}^{M}=0.1$ and all other dispersion hyperparameters set to zero ( $95 \%$ confidence interval). 
The ML-SROM allows statistical dispersion levels to be controlled within each subspace. A sensitivity analysis of the ML-SROM with respect to the dispersion parameters is carried out. We are interested in performing a sensitivity analysis of frequency response functions for structural accelerations over the frequency band $\mathcal{B}=[10,1500]$ Hz. To do so, the modulus in $\mathrm{dB}$ of the random normal acceleration in one point of the car (that is distant from the excitation point) is calculated using Eq. (28) with $n_{\text {sim }}=300$ Monte-Carlo realizations. The $95 \%$ confidence intervals of the random response are plotted:

- in Fig. 1 for $\delta_{\mathcal{L}}^{M}=0.1$ and for all other dispersion hyperparameters set to zero.

- in Fig. 2 for $\delta_{\mathcal{M}}^{M}=0.1$ and for all other dispersion hyperparameters set to zero.

- in Fig. 3 for $\delta_{\mathcal{H}}^{M}=0.1$ and for all other dispersion hyperparameters set to zero.

For only nonzero $\delta_{\mathcal{L}}^{M}$, the confidence interval is larger in the low-frequency band whereas it is very thin elsewhere. For only nonzero $\delta_{\mathcal{M}}^{M}$, the confidence interval is larger in the mid-frequencies although the dispersion also propagates around. For only nonzero $\delta_{\mathcal{H}}^{M}$, the confidence interval is larger in the high-frequency band although it is also large in the mid-frequency band. These results tend to agree with the qualitative expectations made hereinbefore. For $\delta_{\mathcal{M}}^{M}=0.1$, the propagation of uncertainties in the high-frequency band is explained by the coupling between subspaces $\mathcal{S}_{\mathcal{M}}$ and $\mathcal{S}_{\mathcal{H}}$, which are not orthogonal with respect to the stiffness matrix.

\section{Conclusions}

A general method has been proposed for the construction of a multilevel stochastic reduced-order computational model devoted to the robust prediction of the frequency response functions of complex linear dynamical systems. The proposed multilevel ROM is based on the nonparametric probabilistic approach. The presence of several structural scales, characterized by the presence of more or less local displacements intertwined with the usual global displacements, and associated with the distinct low-, medium-, and high-frequency bands, induces a heterogeneous variability. Using several orthogonal subspaces each one composed of a particular type of displacements, the multilevel stochastic ROM allows for constructing a finer modeling of uncertainties. We have demonstrated the capability of the method proposed to adapt the stochastic modeling to the level of uncertainties as a function of the frequency bands that can be correlated to the scales of the global and of the local displacements.

\section{Acknowledgements}

This research was supported by Agence Nationale de la Recherche, Contract HiMoDe, ANR-2011-BLAN-00378. The authors thank PSA Peugeot Citroën for providing the car computational model. 


\section{REFERENCES}

[1] R. Ohayon, C. Soize, Advanced Computational Vibroacoustics - Reduced-Order Models and Uncertainty Quantification. Cambridge University Press, 2014.

[2] L. Meirovitch, Dynamics and Control of Structures. Wiley, 1990.

[3] J. Argyris, H.P. Mlejnek, Dynamics of Structures. North-Holland, 1991.

[4] R.R. Craig, A.J. Kurdila, Fundamentals of Structural Dynamics. Wiley, 2nd Edition, John Wiley and Sons, 2006.

[5] C. Soize, A. Batou, Stochastic reduced-order model in low-frequency dynamics in presence of numerous local elastic modes. Journal of Applied Mechanics Transactions of the ASME, 78(6), 061003-1 to 9, 2011.

[6] A. Batou, C. Soize, Uncertainty quantification in low-frequency dynamics of complex beam-like structures having a high-modal density. International Journal for Uncertainty Quantification, 1(1), 431-451, 2013.

[7] A. Arnoux, C. Soize, A. Batou, L. Gagliardini, Reduced-order computational model for low-frequency dynamics of automobiles. Advances in Mechanical Engineering 310362, 1-12, 2013.

[8] O. Ezvan, A. Batou, C. Soize, Multilevel reduced-order computational model in structural dynamics for the low- and medium-frequency ranges. Computer and Structures, 160, 111-125, 2015.

[9] C. Soize, Random matrix theory for modeling uncertainties in computational mechanics. Computers Methods in Applied Mechanics and Engineering, 194(1216), 1333-1366, 2005.

[10] C.E. Shannon, A Mathematical Theory of Communication. Bell Syst. Tech. J., 27, 379-423 and 623-659, 1948.

[11] E.T. Jaynes, Information Theory and Statistical Mechanics. Phys. Rev., 106(4), 620-630 and 108(2), 171-190, 1957.

[12] R. Rubinstein, Simulation and the Monte Carlo Method. John Wiley and Sons, 1980. 